\section{EMF-cancer link still murky}

\section{Washington}

THE mystery surrounding the connection between electromagnetic fields (EMFs) and cancer deepened last week. In a longawaited development, epidemiologist John Peters from the University of Southern California (USC) released preliminary results from a case-control study of 232 young leukaemia victims, but the data raised more questions than they answered. They implied that leukaemia risks are correlated to EMF exposure, and that they are not, depending on the how the exposure is estimated.

During the past several years, a series of epidemiological studies has raised concern about the possible health risks of EMFs generated by power lines and electrical appliances. Several researchers have found increased rates of brain cancer and leukaemias among electrical workers and others exposed to EMFs in their jobs.

But the greatest worries have surrounded the persistent claim that children exposed to high levels of magnetic fields may be two or three times more likely to contract leukaemia than those not exposed.

To test this claim, Peters examined 232 cases of leukaemia in children 10 years old or younger from 1980 to 1987 in Los Angeles County. For each of the 232, he chose a control of the same age, sex and race. He collected an array of information on the 464 cases and controls, including medical histories of parent and child, residential histories and job histories of the parents. He assessed the EMF exposure of each child in a variety of ways: spot measurements inside and outside the home, continual measurements in the child's bedroom over periods of 24 to 72 hours and a method of "wire coding" that estimated the level of EMFs in the home by examining the types of electric power lines that ran nearby.

The USC epidemiologist presented his preliminary analysis of these data on 7 February to an EMF workshop in Carmel, California, sponsored by the Electric Power Research Institute (EPRI).

Peters found no relation between measured electric field exposure and leukaemia risk and only a small, not statistically significant, correlation between 24-hour magnetic field measurements and leukaemia risk.

He did, however, find a statistically significant correlation between EMF exposure as estimated by wire coding, and leukaemia risk. Children who lived in homes in the highest code category were 2.5 times as likely to develop leukaemia as those in the lowest-level homes.

The workshop participants were puzzled by Peters' data, said Patricia Buffler, the Univerity of Texas Medical School epidemiologist who organized the meet- ing. Why should the wire codes correlate measured electric and magnetic fields in the homes do not? But perhaps the most puzzling thing is that Peters' results fit into a consistent pattern of such data.

The earliest study to have found a connection between EMFs and childhood cancer was performed in Denver, Colorado, by Nancy Wertheimer and Ed Leeper in 1979. Because the two could not afford devices to measure EMF fields directly, they developed a system by which they estimated the EMF levels near power lines according to the type of line. (Depending on a line's place in the electrical distribution system, it will carry from 115 volts to several hundred kilovolts.)

Working from this wire coding system, Wertheimer and Leeper claimed that children who lived in homes exposed to highlevel EMFs developed leukaemia and lymphomas up to three times as often as children who lived in homes with low-level fields. The study was generally discounted, however, first because biologists knew of no mechanism by which such

\section{Danger from electrical appliances?}

BLACK-AND-WHITE televisions and hair dryers were correlated with increased cancer risk in epidemiologist John Peters' case-control study of 232 victims of childhood leukaemia, but the implications of that finding are still unclear.

Peters asked the parents of those 232 children and of 232 controls to report the children's use of 13 different electrical appliances, such as microwave ovens and curling irons. In most cases, appliance use correlated with some increased risk of leukaemia, Peters told a workshop sponsored by the Electric Power Research Institute, and the risk was statistically significant for black-and-white televisions and hair dryers.

One possible explanation for the results could be 'recall bias' - parents with sick children might be more likely to recall use of appliances perceived to be dangerous than parents of healthy children. However, Peters pointed out to the workshop that if this recall bias was at work, it did not create a correlation between cancer risk and microwave ovens, an appliance that many people might perceive as dangerous. And the use of colour televisions did not have as large a risk factor as did black-andwhite televisions - another factor that would be hard to explain with recall bias. with increased cancer risk when the fields could cause or promote cancer and, second, because they had not made direct measurements of the electric and magnetic fields inside the children's homes.

Thus it came as a surprise to many researchers when David Savitz at the University of Colorado Medical School essentially replicated the WertheimerLeeper findings in a study published in the well-respected American Journal of Epidemiology in 1988.

In a survey designed to avoid many of the shortcomings of its predecessor, Savitz found that children who were exposed to high-level EMFs in their homes were twice as likely to develop cancer as those whose homes had lower exposure - as estimated by a wire-coding system similar to that of Wertheimer and Leeper.

Savitz, however, also made direct spot measurements of the electric and magnetic fields in the homes and found a curious anomaly. Although the spot measures of the electric and magnetic fields generally agreed with the estimates obtained by wire coding, the correlation between cancer risk and measured EMF strength in the home was not nearly as high as the correlation between cancer risk and the EMF levels as estimated by wire coding. At the time, Savitz speculated that the wire coding might be giving a more accurate measure of a child's long-term EMF exposure than the one-time spot measurements. If true, then exposure to high levels of electric or magnetic fields might indeed be hazardous to a child's health.

So Peters monitored EMFs in children's homes over 24- and 72-hour periods in hopes of erasing the discrepancy between wire coding and measured EMF levels. But it just got worse. Peters found that "the wire codes and the measured fields did not correlate very well", Buffler said, and the wire codes, but not the measured fields, correlated with cancer risk.

What's going on? Peters would not talk to the press, but Buffler said discussion at the meeting centered on two possibilities. First, some types of EMF exposure may indeed lead to a higher leukaemia risk, and the wire coding may be a more sensitive indicator of exposure than even 24hour measurements; in this case, it will be important to work out what the wire coding is picking out that is missed by direct measures of the fields inside the homes. On the other hand, it is possible that the correlation between wire coding and leukaemia risk may be merely an artefact, albeit a stubborn one.

"People are not yet willing to believe that it's a real effect", Buffler said. But until someone can explain why one measure of EMF exposure gives one answer, and a second measure gives another, the worry that EMFs can promote cancer will remain. 